\title{
How do common investors behave? Information search and portfolio choice among bank customers and university students
}

\author{
Marco Monti • Riccardo Boero • Nathan Berg • \\ Gerd Gigerenzer • Laura Martignon
}

Received: 16 April 2012/ Accepted: 11 June 2012/Published online: 10 July 2012

(C) Springer-Verlag 2012

\begin{abstract}
Bank customers are not financial experts, and yet they make high-stakes decisions that can substantively affect personal wealth. Sooner or later, every individual has to take relevant investment decisions. Using data collected from financial advisors, bank customers and university students in Italy, this paper aims to reveal new insights about the decision processes of average non-expert investors: their investment goals, the information sets they consider, and the factors that ultimately influence decisions about investment products. Using four portfolio choice tasks based on data collected directly from financial advisors and their clients, we find that most subjects used a limited set of information, ignoring factors that conventional economic models usually assume drive investor behavior. Furthermore, we suggest that non-compensatory decision-tree models, which make no trade-offs among investment features, are parsimonious descriptions of investor
\end{abstract}

\footnotetext{
M. Monti · G. Gigerenzer

Max Planck Institute for Human Development-Berlin, Lentzeallee 94, 14195 Berlin, Germany

e-mail: monti@mpib-berlin.mpg.de

G. Gigerenzer

e-mail: gigerenzer@mpib-berlin.mpg.de

R. Boero $(\bowtie)$

Department of Economics and Public Finance, University of Torino,

C.so Unione Sovietica 218/bis, 10134 Turin, Italy

e-mail: boero@econ.unito.it

\section{N. Berg}

School of Economic, Political and Policy Sciences, University of Texas-Dallas, 800 West Campbell Road, GR 31, Richardson, TX 75080-3021, USA

e-mail: nberg@utdallas.edu

L. Martignon

Institut für Mathematik und Informatik, University of Education-Ludwigsburg,

Reuteallee 46, 71634 Ludwigsburg, Germany

e-mail: martignon@ph-ludwigsburg.de
} 
behavior useful for improving the organization of financial institutions and in policy contexts alike.

Keywords Behavioral finance - Investment decision - Portfolio composition · Non-compensatory heuristic · Recognition heuristic · Ecological rationality

\section{Introduction}

This study seeks to describe the decision-making processes of bank consumers who make portfolio decisions in consultation with financial advisors employed by banks and to compare their choices with the ones of completely inexperienced investors such as undergraduate students. In a first, hypothesis-finding step, we collected qualitative and quantitative data using in-depth interviews with both financial advisors and their clients concerning the typical range of investment alternatives presented to consumer investors. Interviews and survey data also provided quantitative descriptions of the typical number of items in investors' consideration sets and the investment features (i.e., information) that investors typically ask for and are supplied with by investment advisors. In a second, hypothesis-testing step, we used the results of the interview and survey data to construct a laboratory-type experiment consisting of four portfolio choice tasks designed to match the investment environment that actual bank customers face.

Thanks to an unusually cooperative experimental partner, a chain of regional banks in Italy in the suburbs of Trento, we were able to conduct these investment task experiments using random samples of actual bank customers and conduct them on-site inside the banks at which these same customers have investment accounts. We think this provides an unusually high degree of match between the subject population and laboratory environment, on the one hand, and the real-world investment behavior of primary scientific interest on the other.

Furthermore, we replicated the experiment in a university computer lab with random samples of undergraduate students: in this way we test the results obtained so far on non-experts' investment choices and we investigate the potential role of experience.

There are five main questions we seek to address: (1) What information do common investors need before making high-stakes investment decisions? (2) Given a large field of information about different investment alternatives and their features, how do common investors search for information? (3) Even when the size of the choice set is modest, can we describe patterns of information search and information usage to reveal how consumers eliminate investments from consideration? (4) Is there confirmatory evidence of pairwise comparison of common investment features consistent with the widespread assumption that consumers rank items in their choice sets by product features? (5) If not, can we specify a descriptive model informed by empirical data that better reveals patterns of information search and the decision processes actually used by investors?

In conclusion, answering the questions we are proposing has some important consequences for both financial institutions and investors, opening the possibility to 
improve the resilience of the economy towards recurrent shocks. In fact, it is our strong belief that hypotheses which identify cycles of over and under risk-exposure and confidence of economic actors as relevant causes of recurrent crisis in financial markets are rightly focused. In particular we claim that, although not the only cause of those phenomena, the imperfect match of investors' preferences in financial markets strongly diminishes the capability of the system to adapt and learn. As a logical consequence of this claim, we believe that misunderstandings about investors' decision making lead not only to poor customers' satisfaction and inefficient organization of financial institutions but also to endemic financial instability.

Yee et al. (2007) show that consumer choice is sometimes better described and predicted by lexicographic, or non-compensatory, decision tree models rather than linear models (conjoint analysis) which are standard in marketing research and economics. We use this insight as motivation to fit non-compensatory decision trees and compensatory tallying rules to portfolio choice data, comparing their predictive accuracy with larger linear models that simultaneously apply weights to all investment features to predict investor behavior. ${ }^{1} \mathrm{We}$ also borrow the technique of process tracing from psychology to filter the data in greater detail in hopes of revealing more about investors' decision process.

One motivation for the approach taken in this study is that improved veridical descriptions of decision-making process which go beyond the as-if methodological goal of predicting decision outcomes would be a substantive advance for descriptive behavioral science. Perhaps just as compelling, especially in light of the institutional failures surrounding recent financial market crises whose consequences continue to play out as we write, these descriptions of decision-making process should facilitate the design of improved institutions that are better matched to the decision-making processes commonly found among bank consumers. Because firms may have incentives to offer smaller ranges of choices to retail (versus online) customers (Lynch and Ariely 2000), this study's rich data, drawing on retail branch bank customer decisions and information collected from those bank employees who dispense investment advice, provides a unique view into retail banking as experienced by consumers.

We would contend that mistaken models of the investor's mind underlie a wide variety of real-world problems, from faulty risk models used by ratings agencies on mortgage-backed securities to misguided marketing campaigns that fail to successfully launch new investment products to consumers. Being well informed about decision process - not merely decision outcomes-matters greatly.

A growing literature in economics and psychology shows that decision makers often do not incorporate all available information into their decisions, even when

\footnotetext{
${ }^{1}$ Linear models of investor behavior, such as those derived from expected utility maximization with a mean-variance expected utility function, assume that all features of each investment alternative are weighted and summed (and possibly transformed by a monotonic function). In contrast, noncompensatory models have a fixed hierarchy of investment features. One investment feature (e.g., high risk) can be enough to entirely discard an alternative from the investor's consideration set, without the possibility of compensation (e.g., standard models typically assume that, no matter how high the risk, this negative can be compensated with sufficiently large positives such as high expected return). Just as Yee et al. (2007) found that consumers can effectively manage large choice sets by quickly shrinking the items in consideration using a single product feature, we consider models which allow data to reveal this kind of fast reduction of choice sets by lexicographically ruling out alternatives based on one undesirable feature.
} 
information is statistically valid, non-redundant (i.e., non-collinear with other predictors), and costless to acquire (Balzer et al. 1989; Chewning and Harrell 1990; Lee and Lee 2004; Berg and Hoffrage 2008). Loewenstein (2006) illustrates the more general point about mismatch between models and underlying behavioral processes that, paradoxically, can get worse when modelers try to introduce new aspects of realism to their models.

We try to demonstrate a technique, implemented in the case study presented here of Italian bank customers (who are typical in that they possess no expert credentials or specialized academic training in finance) and undergraduate students (who do not possess experience in investments but who have received a basic training in finance), to reveal how average investors search for information and map that information into portfolio decisions.

If information search is limited rather than exhaustive, how are pieces of information that are considered mapped into actual decisions? Gigerenzer et al. (1999) put forward a positive theory regarding simple and information-frugal decision rules that have attractive properties (e.g., accuracy in prediction tasks, as in Gigerenzer and Brighton 2009; Martignon and Laskey 1999, and Gigerenzer and Goldstein 1996) and solid empirical support based on lab experiments.

Fast-and-frugal heuristics can make accurate predictions in a variety of domains (Gigerenzer et al. 1999). Information-frugal models classify and infer with only limited knowledge and computational effort, using a strict subset of the available information. Some of the heuristics we consider, as in Yee et al. (2007), make no trade-offs between investment features. Instead, they process sequentially and lexicographically one investment feature at a time. Under certain circumstances, such heuristics can be as accurate as models based on standard definitions of "rational choice" in economics (e.g., weighted linear models and Bayesian approaches that take all features and their entire correlation structure into account, assuming that decision makers somehow have access to all the relevant parameters-see Martignon and Laskey 1999, and Gigerenzer et al. 1999).

The paper proceeds as follows. Section 2 describes the experimental design, the samples from which the data were collected, and details of the four experimental tasks tailored to match Italian bank customers' investment environment. Sections 3 and 4 present the empirical results, describing a number of patterns in subjects' search for information and prediction models for consumers' ranking of investments and portfolio choice. Moreover, Sects. 3 and 4 fit Markov transition matrices for information search, response-time data, process tracing, non-compensatory heuristic models of investment choice, and linear models that attempt prediction of consumer's choice of investment products actually offered to them by banks. Finally, Sect. 5 concludes with a discussion and interpretation of the empirical results.

\section{Methods}

The research project was developed in three phases. We first collected interviews from 20 professional financial advisors and then from 80 customers of an Italian mutual bank. The interviews focused on interactions between advisors and their 
clients with active investment accounts. An important focus was the investors' experiences communicating with advisors about risk, and the information they valued (or not) when allocating investment funds to different investment products. Fifteen customers from the sample were also invited to participate in four investment choice tasks. The same tasks were then presented to undergraduate students. We analyze this experimental data at the aggregate level comparing the two subjects' samples and then investigate within-subject treatment effects across the four investment tasks. The four investment tasks systematically modify and test one real-world aspect of advisor-client communication.

\subsection{Participants}

Subjects were customers of an Italian mutual bank and students attending the courses of an undergraduate program in Business Administration at the University of Torino, Cuneo campus. Fifteen bank customers and 56 undergraduate students participated in a sequence of four experimental computer-interactive tasks, implemented using an original computer interface.

A mutual bank is a nonprofit financial institution whose aim is to support the economic well-being of the people living in a specific region. We selected this type of bank because its financial advisors do not earn commissions and face little or no pressure to sell investment products. Given that their incentives are likely much more closely aligned with investors' interests than those in other institutional arrangements, the mutual bank offers what is perhaps the most conducive environment one might hope for in terms of clear and effective communication between financial advisors and their clients. This environment, we hope, provides a best-case scenario for learning about the information consumers want and use, and which elements of financial communication work and do not work from the consumer perspective, without the confounding influence of possibly adversarial incentives.

Customers were randomly selected from the bank database, which contains all active customers. When randomly drawing customer/subjects, we imposed a minimum deposit threshold of 40,000 Euros to help ensure that investment decisions we observed were relatively high stakes.

Students were mainly in the second year $(94.6 \%, 3$ subjects were at the third year) of their program and all regularly attended obligatory lectures, in particular "Finance for business management" and "Macroeconomics," where they received basic training in concepts such as bonds, stocks, and investment funds and on how to compute their return rates. Students were, at the time of experiment (in May 2010; average age: 21.1), inexperienced in investments, having never made investment choices $(100 \%)$ but experienced in using a bank current account for managing their liquidity $(100 \%)$. Finally, 34 of the participating students were male $(61 \%)$ and 22 female (39\%).

\subsection{Design overview}

We designed four hypothetical investment tasks to keep track of financial consumers' information search and actual decision making in the field. The bank 
customers did not receive rewards for completing the task, while the undergraduate students did. We discuss the rationale for this approach below.

To make the tasks as close to a real investment environment as possible, the information setup of the investment tasks relied partially on the investment features that financial advisors commonly present to their customers, as pointed out during the interviews we conducted. We used information collected from the financial advisors to calibrate investment features presented in the experiments that realistically match the features of investment options commonly presented to customers.

We investigated subjects' decision strategies according to three main characteristics: (1) the amount of information subjects use when making financial decisions, (2) the type of information (features or cues) they consider before choosing their investments, and (3) the decision process they follow when making decisions.

Computer-administered investment tasks were carried out with bank customers at different bank branches in and around Trento, Italy. An experimenter read the instructions to each participant out loud. Each session was run with a single bank customer. The average session lasted $60 \mathrm{~min}$ for the experimental tasks and $15 \mathrm{~min}$ for a post-experiment interview. Investors participated voluntarily in the experiments and were not remunerated. Most subjects showed great enthusiasm and gave statements indicating that they viewed their participation as a contribution improving the quality of service at their mutual bank.

Investment tasks were conducted on a touch-screen-based interface programmed in Java. We designed this computer interface specifically to facilitate interaction of a wide range of consumers with different demographic characteristics. In particular, we wanted an experimental interface with large, easy-to-read, icons that would work well for older investors. Each subject was placed in front of the touch-screen and trained extensively in a one-on-one teaching format on how to operate the computer and the details of all tasks. All experimental decisions were recorded automatically by a PC running a Java Virtual Machine, and interviews were recorded as digital audio files that were later transcribed.

Each experiment was composed of four different tasks. In Task 1, subjects were asked to choose between two investments based on risk, the availability of a fixed return rate, investment time horizons, management fees, liquidity, and costs for their redemption. In Task 2, the number of investments was expanded to six, and we observed the information subjects chose to look up, their sequence of information search, and their most preferred investment choice. In Task 3, instead of choosing from a stated menu of investment choices, subjects were asked to design their preferred investment portfolio based on investment labels indicating general categories of asset classes (e.g., equity, treasury bills, other fixed income, real estate). Labels such as "treasury bills" are typically used by investment advisors as benchmarks when discussing risk, serving as the closest real-world analog to the risk-free returns that appear in many theoretical models of portfolio choice such as CAPM. We used these labels to better understand their effects, given that they are commonly used by investment advisors in consultations with bank customers. In Task 4, individuals were asked to repeat the asset allocation decision from Task 3 , this time using only individual investment features but no labels for asset categories. 
This design allows for within-person analysis of the effect of labels on portfolio choice. In all experiments, we randomized the order in which investment features were presented. The goal was to avoid influencing information search by presenting information in a fixed order.

For conducting the experiment with university students, we implemented the interface outlined above in z-Tree (Fischbacher 2007) and ran the experiment simultaneously in two rooms of a computer lab of the University of Torino, Italy, controlling for a complete absence of communication between subjects.

All the investment features we presented to our subjects, both bank customers and university students, were according to six "investment models" we observed in the field. These were chosen based on different data collected about representative menus facing bank customers at the banks we studied. All six investment alternatives are those that are typically available in banks: bank accounts, bonds issued by the bank, bonds issued by the government (i.e., the Italian equivalent of US treasury bills), insurance products, balanced mutual funds (with a roughly 50-50 portfolio in corporate bonds and blue chip stock equities), and stocks (a valueweighted index of Italy's largest 40 publically traded companies trading in markets with euro-dominated shares). According to the actual characteristics of those investments we assigned values to the features of our "investment models" (e.g., stocks have a high risk value, governments bonds have low risk, etc.; see Fig. 3 for full details). Throughout the experiment, we presented combinations of these features.

The following four sub-sections describe each task presented to bank customers, and last one presents the modifications we implemented to the experimental design in order to obtain comparable results with undergraduate students, that is, the incentive mechanism we adopted.

\subsection{Task 1: Binary investment choice}

When asked to choose between two investments, subjects were invited to search for information presented in a $2 \times 6$ matrix. Each of the two rows represented one of the two investment alternatives. Each of the six columns contained information about one of the following investment features-risk, time horizon, management costs (intermediary fees), liquidity, costs for redeeming the invested capital before the investment natural end, and coupon rate (Fig. 1). The investment features, about which each column provided information, were labeled, but the information in those information matrix cells was hidden, allowing us to measure which information cells were "looked up" by subjects and in which order. ${ }^{2}$ Bank customers did not face monetary costs of information. The experimental design does, however, impose a limit of six as the maximum number of investment features that can be looked up (out of 12 that could be looked up in the absence of any limit) to generate

\footnotetext{
2 These terms are translations of the Italian terms used by the banks' financial advisors. Their imprecision would likely make any student of financial economics blush or, better still, demand more specificity. What, for example, does "risk" mean? Nevertheless, our goal was to calibrate all experimental details to the actual investment environment faced by the consumers who served as subjects. Thus, the terms are regrettably vague: rischio, durata, costo, liquidabilità, spese di uscita and interessi.
} 


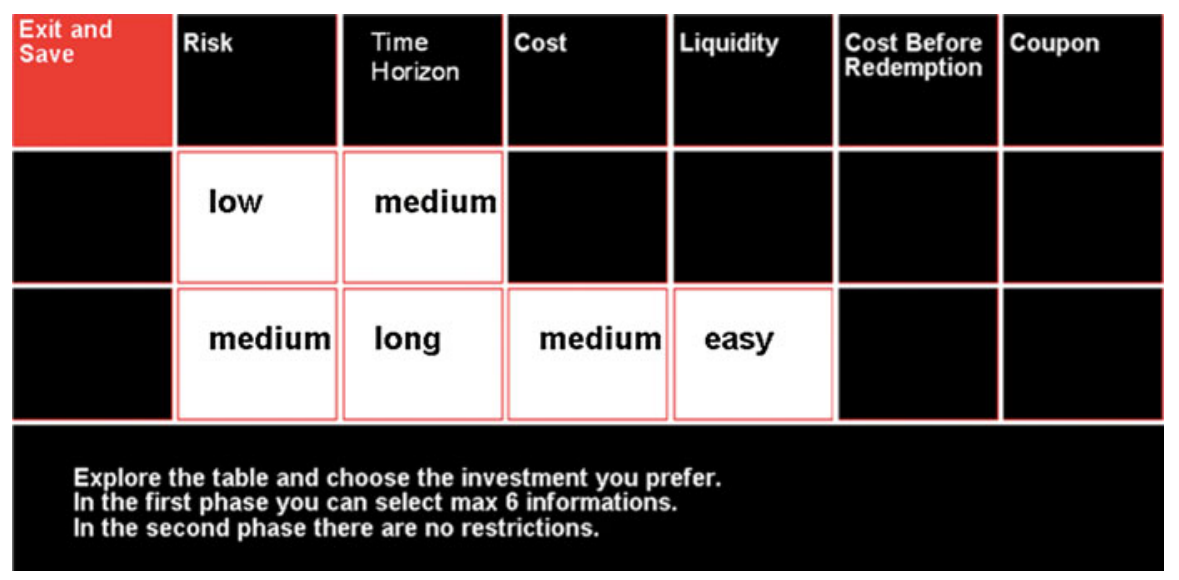

Fig. 1 Task 1, choosing between two investments

meaningful opportunity costs associated with each investment feature that is looked up. ${ }^{3}$

Each task began by presenting a black matrix on the screen, initially hiding all information about investment features. Each subject was asked to explore those features that they considered helpful for identifying their preferred investment. Figure 1 shows a typical screen for Task 1 after six pieces of information about four investment features have been looked up. A decision is about to be made.

\subsection{Task 2: Extended information search}

Task 2 forces the same subjects to confront an investment environment with a larger choice set and considerably more information that could be looked up. The investor's choice set now contains all six alternatives.

Figure 2 depicts this larger information field, consisting of six investment alternatives. The order in which investment features were presented to subjects was randomized in each trial. Therefore, the order of the columns of Fig. 2 differed for each subject.

The screenshot in Fig. 2 shows a subject who has already looked up 14 out of the 36 available pieces of information. Subjects were asked to look up only the information they considered necessary for choosing their most preferred investment portfolio. Once again, the task began by presenting an information matrix in which all investment features were initially blacked out. No restrictions on the number of investment features that could be looked up were imposed.

\footnotetext{
3 The maximum number of six investment features that can be looked up actually comes from information collected from investment advisors. They told us that, due to time constraints and limitations of customers' understanding of and capacity to absorb investment information, they commonly consider the upper limit to be six pieces of information.
} 


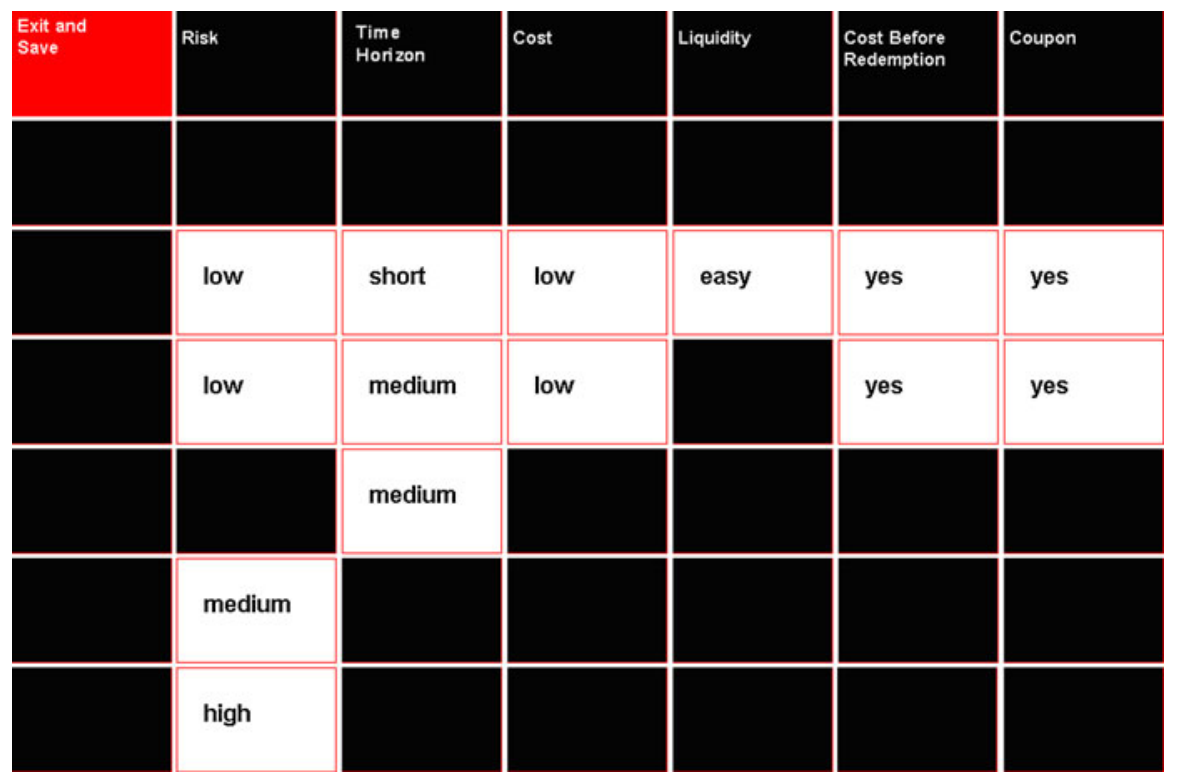

Fig. 2 Task 2, extended information search

\subsection{Task 3: Portfolio choice with asset class labels}

Unlike Tasks 1 and 2, subjects in Task 3 were provided with the full information matrix revealing all investment features from the beginning. Their task was to choose an investment portfolio by writing down portfolio weights (i.e., a list of six numbers summing to 100 that represent the percentages of the investor's wealth allocated to the six investment categories in the rows of Fig. 2).

Figure 3 illustrates the screen that elicits subjects' portfolio weights. The first column contains the name (i.e., label) of each investment. The white boxes show the investment features. The last column contains the investors' portfolio weights (sometimes referred to as allocation decisions). Buttons on the boxes where subjects entered portfolio weights adjusted in 5 percentage-point increments. The heading of the last column dynamically displays the percentages of investor wealth allocated to each row, which corresponds to an investment or investment class serving as one element in the investor's portfolio. The screen shot shown in Fig. 3 depicts an investor's portfolio choice in mid process. Only $70 \%$ of the investor's wealth has been allocated so far. The investor then continues adjusting the portfolio weights until a full $100 \%$ has been allocated across the six rows show in the final column.

\subsection{Task 4: Zero information portfolio choice}

Task 4 was identical to Task 3 except for the first column. The first column displayed asset class labels in Task 3. In Task 4, those labels are hidden, as shown in Fig. 4. No other changes were introduced apart from a random reshuffle in columns 


\begin{tabular}{|c|c|c|c|c|c|c|c|}
\hline $\begin{array}{l}\text { Next Trial } \\
\text { or Exit and } \\
\text { Save }\end{array}$ & Liquidity & Coupon & $\begin{array}{l}\text { Cost Before } \\
\text { Redemption }\end{array}$ & Risk & $\begin{array}{l}\text { Time } \\
\text { Horizon }\end{array}$ & Cost & \begin{tabular}{|l} 
Split the \\
Pie \\
$70 / 100$
\end{tabular} \\
\hline $\begin{array}{l}\text { Insurance } \\
\text { with Guaranteed } \\
\text { Capital }\end{array}$ & difficult & no & yes & low & medium & high & $5 \%$ \\
\hline $\begin{array}{l}\text { Mutual Bank } \\
\text { Bonds }\end{array}$ & easy & yes & yes & low & short & low & $10 \%$ \\
\hline Bank Account & easy & yes & no & low & short & low & $15 \%$ \\
\hline $\begin{array}{l}\text { Government } \\
\text { Bonds }\end{array}$ & easy & yes & yes & low & medium & low & $20 \%$ \\
\hline $\begin{array}{l}\text { Balanced } \\
\text { Mutual Funds }\end{array}$ & easy & no & no & medium & long & medium & $20 \%$ \\
\hline Stocks & easy & no & no & high & long & medium & $0 \%$ \\
\hline
\end{tabular}

Fig. 3 Task 3, investment portfolio selection

\begin{tabular}{|c|c|c|c|c|c|c|c|}
\hline $\begin{array}{l}\text { Next Trial } \\
\text { or Exit and } \\
\text { Save }\end{array}$ & Cost & Risk & $\begin{array}{l}\text { Cost Before } \\
\text { Redemption }\end{array}$ & Liquidity & $\begin{array}{l}\text { Time } \\
\text { Horizon }\end{array}$ & Coupon & $\begin{array}{l}\text { Split the } \\
\text { Pie } \\
50 / 100\end{array}$ \\
\hline & medium & medium & no & easy & long & no & $0 \%$ \\
\hline & low & low & yes & easy & medium & yes & $10 \%$ \\
\hline & medium & high & no & easy & long & no & $15 \%$ \\
\hline & low & low & no & easy & short & yes & $25 \%$ \\
\hline & low & low & yes & easy & short & yes & $0 \%$ \\
\hline & high & low & yes & difficult & medium & no & $0 \%$ \\
\hline
\end{tabular}

Fig. 4 Task 4, "Blind" portfolio choice

to re-order investment features. Investors were asked to state their decision processes aloud while the experimenter kept a written protocol; we digitally recorded the descriptions subjects reported while performing the tasks and then summarized them into summary schemes.

\subsection{Changes in experimental design introduced for university students}

As mentioned before, the sample of bank customers received no monetary incentives in the experiment, the hypothetical investment choice being a familiar one to them. For the sample of university students that approach could not be followed for obvious reasons: lacking experience in the field, they were absolutely unfamiliar with making investment choices. 
Thus we introduced an incentive mechanism by modeling a financial market where the different kinds of investments generated different returns relying on stochastic procedures. The details of the modeling of the considered financial investments are presented in the appendix to this paper, but here it is worth stressing its underlying principles.

In addition to the features of each investment choice explained above, three important features of financial investments needed to be modeled: how investment features translate into monetary earnings, the uncertainty of future events, and the cost of information search.

Taking this into consideration, the rationale of the design is to replicate actual financial investments, and thus different parameters for costs, coupon, etc. are introduced. These parameters are used to compute a payoff function for each investment chosen by subjects. However, with investment choices being subject to uncertainty, two stochastic processes are modeled in the payoff function in order to capture the "risk" and "time horizon" features of investments.

In summary, our undergraduate students knew that the investments they were going to choose led to realistic outcomes subject to uncertain future events. We did not provide the full description of the payoff function we implemented as explained in the Appendix, but we explained the meaning of all investment features and their possible consequences on the payoff.

Finally, taking into consideration the analytic focus on information search (which is investigated in Task 1 and 2), we introduced a cost for information exploration of 6 ECUs (i.e., experimental currency unit) for each piece of uncovered information in Task 1 and of 2 ECUs for each piece of information uncovered in Task 2. In order to avoid confusion about the cost of unveiling information in the two tasks, such information was clearly written in the interface of each task.

The differences in cost between Task 1 and Task 2 were made because of the different availability of pieces of information and of investments on offer. In Task 1 there are only two possible investments and 12 unrevealed features, while in Task 2 these numbers are three times larger; thus we chose to make exploration cost three times less expensive.

Moreover, the exchange rate between ECU and euro was communicated to subjects at the beginning of the experiment and it was set at $1 \mathrm{ECU}=1$ Euro cent. At the end of the short experiment (it lasted about $20 \mathrm{~min}$, including the time for payment), besides paying to subjects a show-up fee of 5 Euros each, the monetary earnings of subjects (i.e., the number of ECUs gained in the experiment and converted at the fixed and known exchange rate) were rounded and subjects received on average 4.50 Euros, with a minimum of 3 and a maximum of 6.5 Euros. The total payoff obtained by students on the basis of their choices was presented at the end of the experiment.

Furthermore, having divided the students' sample into two separate rooms, we took advantage of the possibility to control for the existence of possible sequence effects between Tasks 3 and 4. Thus, half of the students took the experiment in the order Task 1, 2, 3, and 4, and half in the order Task 1, 2, 4 and 3. The results presented in this paper for this sample thus randomize the sequence of the two last 
tasks. Moreover, as for the bank customers, we randomized the order of investment features (i.e., liquidity, cost, coupon, etc.) that students faced.

Finally, while the sample of bank customers was smaller (15 subjects) and Task 1 was repeated on average 4 times by each subject, the sample of students was larger (56 subjects) and Task 1 was repeated three times by each. The remaining tasks $(2,3$ and 4) were performed only once by both bank customers and university students.

\section{Results on information search}

The results are presented in two sections, one concerning the descriptive analysis of subjects' information search behavior and the other concerning the modeling of decision strategies.

In examining the approach followed by investors in searching for financial information, we first considered how much information an investor needed in order to arrive at an investment decision. We investigated the information search processes occurring in both Task 1 (pair-wise investments comparison) and Task 2 (extended information search with an expanded choice set and list of investment features).

In Task 1, $86 \%$ of customers looked at all six pieces of information. In Task 2 , customers considered, on average, less than half of the available information (45\%), revealing a preference for partial rather than full information to act upon. This preference was confirmed by students: in Task 1, $26 \%$ of university students explored 6 or more pieces of information (the mean of explored cues is 4.1 , that is to say the $34.5 \%$ of available information), and in Task 2, they explored on average 12.8 cues, that is to say the $35.6 \%$ of available information.

\subsection{Quantity of look-ups and types of information searched}

Table 1 shows the look-up rate per search for each investment feature in Task 2 . It shows the percentage for all searches, each consisting of a selection of information about six investments that could have been looked up.

As shown in Table 1 on the left, the three main investment features that customers focused on were risk, time horizon, and management costs. Subjects'

Table 1 Look-up rates among all search trials, by investment feature in task 2

\begin{tabular}{llll}
\hline Feature & Look-up rate & Feature & Look-up rate \\
\hline Bank customers & & Undergraduate students & \\
Risk & 76.2 & Risk & 65.8 \\
Time horizon & 48.8 & Coupon & 44.6 \\
Costs & 47.6 & Liquidity & 31.5 \\
Liquidity & 41.7 & Time horizon & 27.7 \\
Coupon & 39.3 & Cost & 24.7 \\
Cost before redemption & 26.2 & Cost before redemption & 12.8 \\
Mean & 46.6 & Mean & 34.5 \\
\hline
\end{tabular}


demand for information about the risk feature dominated all other investment features by a sizable margin. In 65 trials, subjects looked up information about risk on more than $75 \%$ of searches, whereas the look-up rate for all other investment features was less than $50 \%$.

Student data largely confirm that for the bank customers. In fact students focused significantly more on the risk feature. The only difference emerging in comparison with customers is the attention to the coupon feature. Students seemed to be more interested in the availability of a predetermined and guaranteed gain. Such a peculiarity is confirmed by the analyses we present throughout this paper but for now it is important to underline that is the only relevant difference we found in the two samples, and that the experiment as a whole confirms that information about risk is searched for in more than $50 \%$ of the cases, while other information features are investigated much less frequently.

\subsection{Temporal analysis of information search}

In Task 1, bank customers sequentially searched for at most 6 different pieces of information while students could search the information space as much as they liked. Table 2 presents the empirical distribution of information look-ups at each of the first search steps, denoted as $t_{1}, \ldots, t_{8}$. This sequential analysis yields results consistent with Table 1 while providing more details about the sequence of search. Risk, time horizon, and management costs tended to be looked up first in the sample of bank customers. Similarly, and consistent with what has been presented before, students look up risk, coupon, and management costs first.

Table 2 Percentage of total look-ups (highest in bold) allocated to various investment features in task 1

\begin{tabular}{|c|c|c|c|c|c|c|c|c|}
\hline Feature & Time $t_{1}$ & & $\mathrm{t}_{2}$ & $\mathrm{t}_{3}$ & $\mathrm{t}_{4}$ & & $\mathrm{t}_{5}$ & $\mathrm{t}_{6}$ \\
\hline \multicolumn{9}{|l|}{ Bank customers } \\
\hline Risk & 89.2 & & 41.5 & 1.5 & 24.6 & & 3.1 & 3.1 \\
\hline Time horizon & 6.2 & & 40.0 & 26.2 & 12.3 & & 6.2 & 6.2 \\
\hline Cost & 0.0 & & 4.6 & 35.4 & 13.8 & & 23.1 & 20.0 \\
\hline Liquidity & 0.0 & & 6.2 & 12.3 & 29.2 & & 21.5 & 16.9 \\
\hline Cost before redemption & 1.5 & & 3.1 & 7.7 & 10.8 & & 32.3 & 16.9 \\
\hline Coupon & 3.1 & & 4.6 & 16.9 & 9.2 & & 10.8 & 30.8 \\
\hline Feature & Time $t_{1}$ & $\mathrm{t}_{2}$ & $\mathrm{t}_{3}$ & $\mathrm{t}_{4}$ & $t_{5}$ & $t_{6}$ & $\mathrm{t}_{7}$ & $\mathrm{t}_{8}$ \\
\hline \multicolumn{9}{|l|}{ Undergraduate students } \\
\hline Risk & 50.3 & 39.2 & 27.1 & 21.6 & 18.0 & 15.9 & 5.0 & 0.0 \\
\hline Coupon & 14.4 & 15.0 & 27.1 & 36.3 & 24.6 & 25.0 & 15.0 & 9.1 \\
\hline Cost & 7.2 & 9.8 & 11.3 & 8.8 & 23.0 & 25.0 & 15.0 & 9.1 \\
\hline Time horizon & 10.8 & 13.7 & 14.3 & 15.7 & 6.6 & 11.4 & 30.0 & 36.4 \\
\hline Cost before redemption & 3.6 & 5.2 & 6.8 & 2.9 & 9.8 & 6.8 & 20.0 & 18.2 \\
\hline Liquidity & 13.8 & 17.0 & 13.5 & 14.7 & 18.0 & 15.9 & 15.0 & 27.3 \\
\hline
\end{tabular}


To make Table 2 clearer, it reveals that at the first opportunity to look up an investment feature $\left(\mathrm{t}_{1}\right)$, bank customers looked up risk in $89.2 \%$ of all trials. At $\mathrm{t}_{2}$, risk was looked up in $41.5 \%$ of all cases, and time horizon $40 \%$. At $\mathrm{t}_{3}$, cost was looked up in $35.4 \%$ of all cases and time horizon $26.2 \%$. These per-period empirical distributions of information search reveal that, at $t_{1}$ and $t_{2}$, risk and time horizon are the most frequently looked up investment features by customers. From $\mathrm{t}_{4}$ onwards, the empirical distribution of look-ups is more evenly distributed over features. It is especially interesting that a predetermined regular gain (i.e., coupon), rather than appearing as the highest priority investment feature for experienced bank customers, is the most frequently looked up feature only in the very last time period, while university students look it up just after risk.

Table 3 presents an estimated Markov transition matrix comprised of the empirical probabilities of moving from one investment feature to another in the bank customers' six-step information search process and in the students' unbounded but expensive information search process. For bank customers at the beginning (start position), the feature most likely to be looked up first is risk (89\% of all first lookups). The feature most frequently looked up following risk is either time horizon (35\% of look-ups following risk) or risk once again (23\% of look-ups following risk). The most frequent look-up after time horizon is either cost (46\% of look-ups after time horizon) or time horizon once more $(16 \%)$.

For students, we observe that risk is the first to be looked up (50\% of all first look-ups at the beginning), followed by cost, coupon, time horizon, and liquidity. Again, what seems to be relevantly different is the focus on coupon, which, however, in the transition matrix appears to happen with highest probability after the exploration of costs.

\subsection{Process tracing of information search}

Following the approach to describing patterns in information search proposed by Payne et al. (2004), we looked at two types of search paths: feature-wise and investment-wise. A feature-wise path corresponds to an investor focusing on just one feature and looking up that same feature across investments. An investmentwise path corresponds to an investor looking up features belonging to just one investment at a time.

For the 14 bank customers, data collected in Task 2 show that 8 of them $(57 \%)$ adopted an investment-wise approach calculated according to Payne's measure. Subjects tended to focus their attention on information pertaining to a single investment at a time. The simultaneous protocol analysis revealed that most customers began searching for information about the investments they already owned in real life (e.g., government bonds [treasury bills] for subject 9, mutual bank bonds for subject 7 , etc.). The other customers searched the available information by adopting mixed strategies: Some of them searched exhaustively for information dealing with risk by adopting a cue-wise approach, while others gathered information across all investments without revealing a predominant approach. 


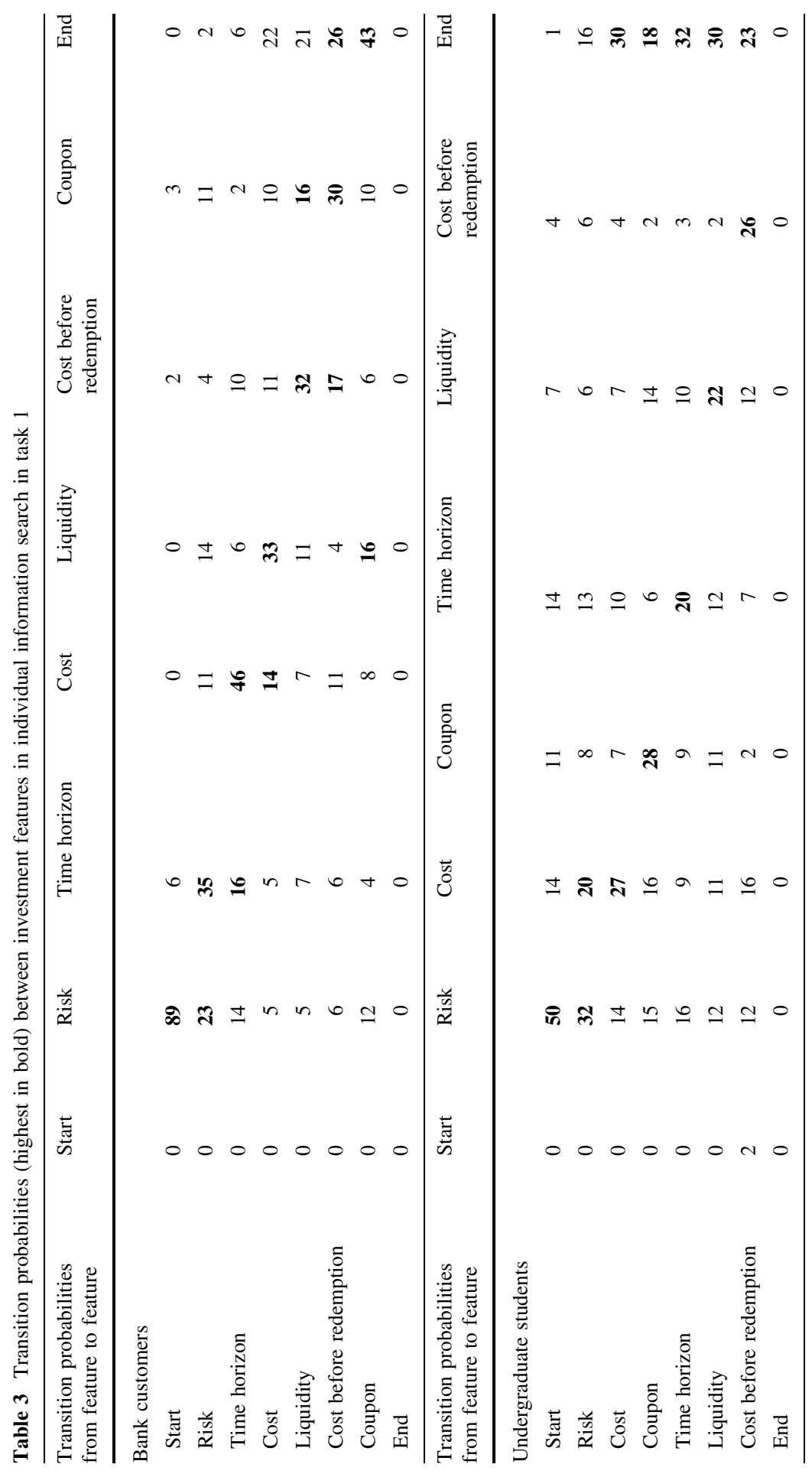


3.4 Overlapping information index and order preservation index

We investigated subjects' information search to answer the following questions: Did customers look at identical information for both investments? And did they search for investment features by following a stable ordering of investment features?

We adopted a within-subject approach by introducing two indices characterizing customers' information search. We noticed that in our experiments, customers did not necessarily collect overlapping information on different investments before making choices. In other words, when considering investments A and B (Task 1), customers did not check the same features for A and B respectively before choosing. Such measures of systematic search, as the two indices we introduced, are not commonly mentioned in the experimental psychology literature, although they are certainly relevant for describing information usage and decision processes which provide an indication of whether neoclassical or heuristic models better describe how customers choose investments.

The two specific measures used here for each participant are: The Overlapping Information Index (OII) and the Order Preservation Index (OPI). We defined the OII as the percentage of identical features looked up for both investments across customers. OPI indicates the percentage of overlapping features searched in identical order. Let us look at two examples. Suppose that a subject explores features of different investment options in the following order: risk, liquidity, then cost, for investment 1 , and cost, risk, then liquidity, for investment 2 . As an alternative example, another subject might search in the following order: risk, cost, and liquidity for investment 1 , and risk, liquidity, and cost, for investment 2. In both examples the OII is $100 \%$, since three out of the three features are looked up for both investments, and for both examples the OPI is $66.6 \%$, because two out of the three overlapping features are looked up in the same order.

Following from above, we classified customers according to OII. We chose a threshold of $50 \%$ to split the sample into low- and high-OII groups:

- High Overlapping Information Index Group (HOI); customers show an OII greater or equal to $50 \%$;

- Low Overlapping Information Index Group (LOI); customers show an OII lower than $50 \%$.

OPI and OII are highly correlated, which means that customers belonging to the HOI group preserve their search order across different searches and focus their attention on a smaller set of financial features (the correlation coefficient between OPI and OII is 0.98 in both subjects' samples). This fact suggests that the selection of the considered information set is strongly linked to individual search processes and with the investment representation space and, thus, with the decision mechanism. By looking at individual values, the aggregate values of which are presented in Table 4, we deduce that a part of our sample looked for coincident information for both investments and followed the same sequential order across cues. 
Table 4 Overlapping information index and order preservation index calculated in task 1

\begin{tabular}{lllll}
\hline Group & Percentage & $\begin{array}{l}\text { Overlapping information } \\
\text { index (in \%) }\end{array}$ & $\begin{array}{l}\text { Mean number of cue } \\
\text { look-ups }\end{array}$ & $\begin{array}{l}\text { Order preservation } \\
\text { index (in \%) }\end{array}$ \\
\hline Bank customers & & & \\
HOI group mean & 53 & 74.7 & 3.7 & 71.8 \\
LOI group mean & 47 & 28.1 & 5.0 & 19.2 \\
Sample mean & 53.0 & 4.3 & 47.3 \\
Undergraduate students & & & 72.5 \\
HOI group mean & 52 & 76.0 & 5.1 & 16.9 \\
LOI group mean & 48 & 17.1 & 3.2 & 45.7 \\
Sample mean & 47.6 & 4.1 & \\
\hline
\end{tabular}

\section{Results on decision strategies}

\subsection{Heuristic model}

This section is devoted to the description of a simple heuristic that describes quite well how bank customers and university students made their decisions. Let us begin by establishing certain notations and introducing some important concepts. We define the cue profile of an investment as a binary vector of $1 \mathrm{~s}$ and $0 \mathrm{~s}$ according to whether cue values are "positive" or not, ordered according to the sequence: risk, time horizon, liquidity, costs (intermediary fees), costs for redeeming the invested capital before the investment natural end, and coupon. Based on the model of takethe-best (Gigerenzer and Goldstein 1996), investment features are all transformed to binary values to simplify their comparison. The convention for assigning the values 1 or 0 to a cue reflects the preferences revealed by customers in their interviews. If for instance, risk is medium or low, it is assigned the value 1, and 0 otherwise (i.e., when risk is unveiled or when its value is high). Similarly, if time horizon is medium or short, its value is 1 , and if cost and liquidity are medium or low, they are also assigned the value 1 . If the investment has no cost before redemption this cue is assigned a 1 , otherwise 0 , and if there are "returns during the holding time" then this cue is assigned a 1 , otherwise 0 .

Based on interviews with financial advisors combined with experimental data on customers' information search, we identified a heuristic that is a hybrid between lexicographic and tallying rules. This hybrid heuristic first examines the one cue that was searched most, namely risk, which lexicographically over-rules all other investment features. For a pair of investments considered to have similar risk, the heuristic ranks the investments by means of a tallying rule depicted in Fig. 5.

Tallying is a heuristic that can be described by a linear model with weights equal to one for each investment feature. In this context, tallying means counting the number of 1's for both investments and choosing the investment with a higher score. For instance, if Investment A has a cue profile, (011111) and B has a cue profile (100000), then B is preferred because the first cue is treated lexicographically. As an example, if $\mathrm{A}$ is an investment with a cue profile (100101) and $\mathrm{B}$ is an investment 


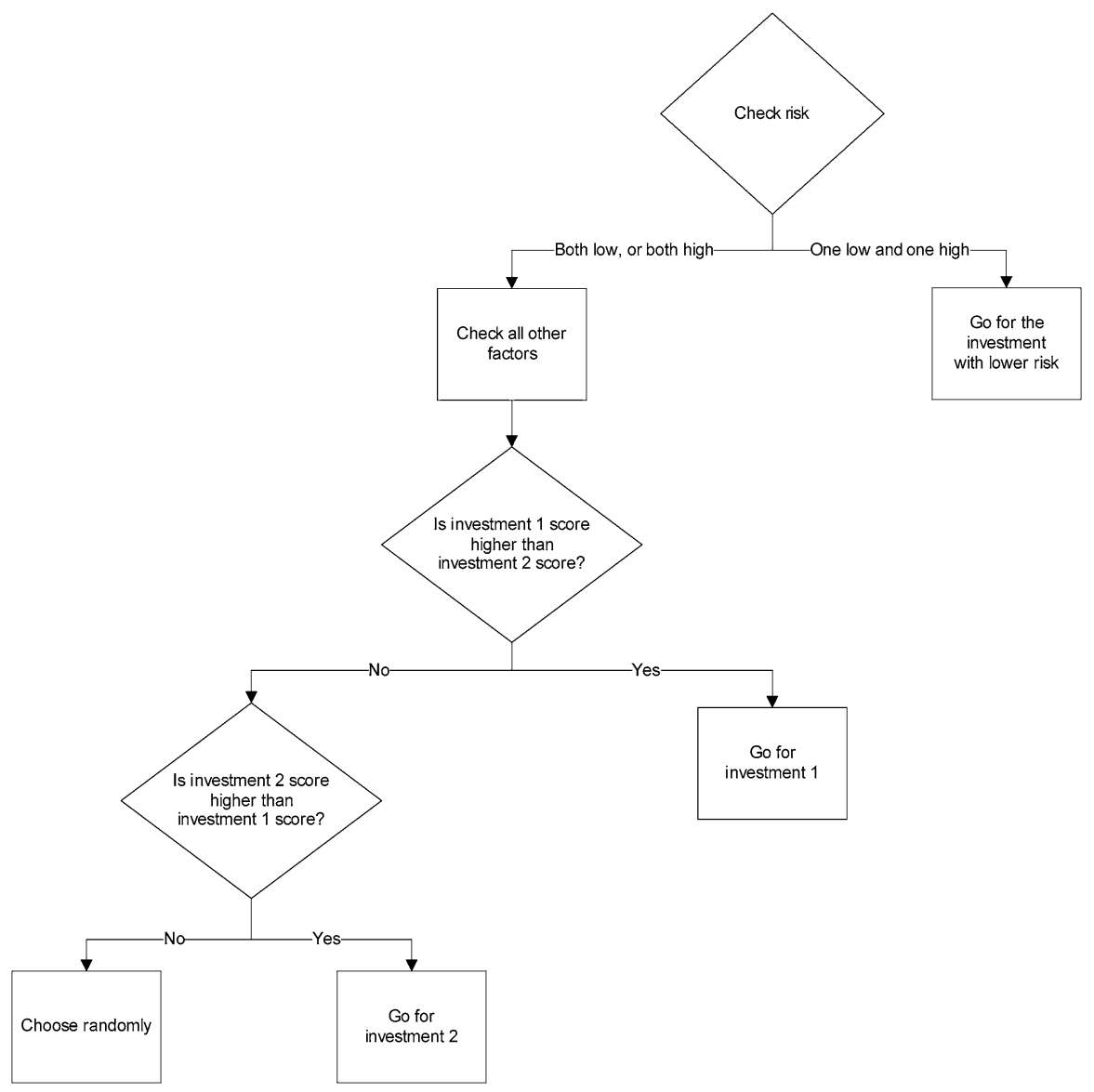

Fig. 5 Fast and frugal tree for binary investment decisions in Table 1

with a cue profile (100100), investment A is chosen over investment B because its profile contains more 1's after the first entry.

How well does the heuristic predict the choices observed in Task 1? Predictions for each subject are presented in Table 5. By fast and frugal tree we mean a tree that has at least one exit at each level; it is "minimal" among trees using all cues, because it has a minimal number of nodes (Martignon et al. 2008).

The tree in Fig. 5 fits about $78 \%$ of the observed investment decisions in Task 1 in the case of the bank customers' sample, and about $74 \%$ in case of the students' sample. The model predicts that for most investors there is no compensating trade-off for high risk investments. High risk investments are eliminated from consideration in the lexicographic formulation. Beyond this lexicographic step that avoids high-risk investments, the model describes investors as simply counting the number of their favorite features on which one investment dominates the other. 
Table 5 Decision heuristic predictions

Scores $(\%)$

$\begin{array}{ll}\text { Bank customers } & \\ \text { Mean } & 78.3 \\ \text { SD } & 21.8 \\ \text { Undergraduate students } & \\ \text { Mean } & 73.8 \\ \text { SD } & 37.9\end{array}$

\subsection{Logit model}

In order to evaluate the heuristic model described above it is possible to refer to two different approaches. The first one is to compare it to models that have a strong theoretical support, in particular those based upon what is usually labeled "rational choice". The second approach is to compare its predictive power with the one of econometric models.

Both approaches do not allow to precisely evaluate the analytic power of the considered model. In other words, often the goodness of a behavioral model can not be evaluated relying only on its predictive accuracy as its capability to capture the reasons and conditions for a specific behavior is extremely important too. However, we leave this latter subtle task to the discussion throughout the paper and to the readers, while in this section we focus on predictive accuracy.

In particular, passing to the comparison of the predictive accuracy of the heuristic model with rational choice models, we can not specify rational choice predictions for the sample of investors: in that case in fact the loose definition of investment choices and the lack of a specific incentive mechanism do not allow the computation of expected returns for each investment choice. On the contrary, in the case of students we can compute expected returns and see how much rational choice predicts the observed data. The maximization of expected returns leads to a correct prediction of the $51.8 \%$ of participants' binary choices, that is a significantly lower rate than the heuristic model and a value similar, in terms of predictive accuracy, to a complete random choice (i.e., $50 \%$ ).

Among the many econometric techniques, we chose logit regression models as the most appropriate to study the problem we are considering here. The multinomial logit model linearly integrates effects of all investment features by taking a weighted average and then transforming (non-linearly) to estimated probabilities. We fit the logit model as a representative of standard linear prediction models as a benchmark against which to compare the fitting accuracy of the heuristic model (Train 2003).

The logit model is widely known and used in countless applications. It assumes that individuals can assign random utility scores to different objects according to the equation $u_{i}=v_{i}+\varepsilon_{i}$, composed of an observable component of utility $v_{i}$ and an unobservable (random) utility component $\varepsilon_{i}$. The assumed decision mechanism is that the individual chooses the object with the highest utility. Hence, under the assumption that $\varepsilon_{i}$ is identically and independently distributed, drawn from the 
Gumbel distribution, the probability of choosing an object is specified by the following functional form: $p_{i}=\exp \left(v_{i}\right) / \sum_{j} \exp \left(v_{j}\right)$, where $j$ indexes all the objects in the choice set (which, in its present application, only includes two objects), and $i$ represents one object of special interest to the modeler in the choice set. As is common practice, we also assume that observable utility is the summation of weighted cue utilities: that is, $v_{i}=\sum_{k} \beta_{k} x_{i k}$. The symbol $x_{i k}$ represents the $k$ th cue of object $i$, and $\beta_{k}$ is the weight parameter that we external observers seek to estimate.

We discretized all cue values in order to normalize the utility value of investment features that were not looked up, even for free, to zero. For example, because the risk feature takes on three levels (low, medium, high), the utility specification coded this information as three dichotomous variables are used, $r_{l}, r_{m}$, and $r_{h}$. When the risk feature is not looked up, all these variables take on the value zero. When the risk feature is looked up and its value is revealed to be low, then the three risk variables are coded as $r_{l}=1, r_{m}=0$, and $r_{h}=0$. Similarly for $r_{m}$ or $r_{h}$. When risk information is looked up, only one of the three risk variables turns on to indicate a value of 1 . As a consequence of this flexible coding that allows for any pattern of marginal utility effects conditional on low, medium, high risk, or no risk information at all, there are three parameters for risk, $\beta_{r l}, \beta_{r m}$, and $\beta_{r h}$.

We chose to infer the model on the bank customers' dataset and in order to avoid as much as possible the problem of non-representative sample due to small sample size, we used a cross validation method. We divided the bank customers' dataset by randomly drawing a $75 \%$ of the sample into the sub-sample for data fitting and a $25 \%$ sub-sample for validation. This procedure was repeated 100 times. Each time, the model was estimated on the fitting sample and validated on the validation sample. After that we also tested the estimated model with students' data.

Table 6 presents average realized values of the estimated coefficients in the linear utility model and counts on the number (out of these 100 prediction trials) in which a particular estimated coefficient was statistically significant. The results provide some evidence about which kinds of information are more important than others, at least in linear prediction. In Table 6, the four most robust cues seem to be high risk, high liquidity, unavailability of cost before redemption, and presence of regular coupon payments. High risk seems to have the largest magnitude impact on utility, which is negative. This is also, of course, consistent with the heuristic model introduced above. The positive sign on the other coefficients suggest that, all else equal, investments with high liquidity, no cost before redemption, and regular coupon payments are preferred, but it is important to note also that several information cues are not relevant for decisions making (i.e., it was impossible to estimate a significant parameter value).

The average percentage of correct hits for the fitting data on bank customers is $84.5 \%$; it is $80.4 \%$ for the validation data, which should be compared with the $78.3 \%$ obtained by the heuristic model. This suggests the full linear model could have over-fitted the data, pointing to another advantage of simpler heuristic models in out-of-sample prediction that has been commented on extensively elsewhere. 
Table 6 Estimation results of the logit model regressed on bank customers data

Mean hit rate for fitting: $84.5 \%$

Mean hit rate in out-of-sample prediction: $80.4 \%$

Mean hit rate in the university students' sample prediction: $76.2 \%$

\begin{tabular}{lcl}
\hline Parameter & $\begin{array}{l}\text { Number of } \\
\text { significant } \\
\text { in } 100 \text { trials }\end{array}$ & $\begin{array}{l}\text { Average coefficient } \\
\text { value in the linear } \\
\text { utility model }\end{array}$ \\
\hline$\beta_{r l}:$ low risk & 20 & 1.3 \\
$\beta_{r m}:$ medium risk & 8 & 2.7 \\
$\beta_{r h}:$ high risk & 72 & -3.3 \\
$\beta_{d s}:$ short time horizon & 16 & 2.7 \\
$\beta_{d m}:$ medium time horizon & 4 & 2.9 \\
$\beta_{d l}:$ long time horizon & 0 & $\mathrm{n} / \mathrm{a}$ \\
$\beta_{c l}:$ low cost & 28 & 1.8 \\
$\beta_{c m}:$ medium cost & 0 & $\mathrm{n} / \mathrm{a}$ \\
$\beta_{c h}:$ high cost & 0 & $\mathrm{n} / \mathrm{a}$ \\
$\beta_{l l}:$ low liquidity & 0 & $\mathrm{n} / \mathrm{a}$ \\
$\beta_{l m}:$ medium liquidity & 0 & $\mathrm{n} / \mathrm{a}$ \\
$\beta_{l h}:$ high liquidity & 100 & 2.1 \\
$\beta_{b u}:$ cost before redemption & 48 & 1.7 \\
unavailable & & \\
$\beta_{b a}:$ cost before redemption & 0 & $\mathrm{n} / \mathrm{a}$ \\
available & & \\
$\beta_{i u}:$ coupon unavailable & 4 & -2.2 \\
$\beta_{i a}:$ coupon available & 54 & \\
\hline & & \\
& &
\end{tabular}

Such a suggestion is confirmed by applying the estimated model to the students' sample: the model fits the $76 \%$ of data, a much lower value than the one obtained with customers' data which is the one on which the estimation has been done. Furthermore, the logit model predictions on the students' sample do not show statistically significant differences with the ones obtained on this sample by the heuristic model (74\%).

\subsection{Role of recognition in portfolio choice}

We now present the role of the investment labels on subjects' choice of portfolio weights in order to investigate the eventual role of familiarity. We collected data on subjects' performance in reproducing the same investments allocation task they performed in Task 3 and 4; the only difference introduced in Task 4 is that we did not provide them with the investment names or labels - just the technical features of the investments. Therefore, financial consumers were still exposed to the same 6 types of investments they encountered in Task 3; namely, stocks, mutual balanced funds, government bonds (treasury bills), savings accounts, mutual bank bonds and insurance with guaranteed capital. The idea was to test how consistent their choices remained when provided with just the investment features and not with their names.

The first result was that for both bank customers and students the availability of labels strongly impacted on portfolio allocations. Given that they paid most attention to risk in the exploration phase, they should have split investments in two 
Table 7 Comparison between portfolio choice across treatments

\begin{tabular}{llll}
\hline & $\begin{array}{l}\text { Weight on risky } \\
\text { assets chosen with } \\
\text { labels }\end{array}$ & $\begin{array}{l}\text { Weight on risky } \\
\text { assets chosen } \\
\text { without labels }\end{array}$ & $\begin{array}{l}\text { Within-subject } \\
\text { percentage } \\
\text { change }\end{array}$ \\
\hline $\begin{array}{l}\text { Bank customers } \\
\text { Mean }\end{array} \quad 21.0$ & 27.0 & 41.4 \\
SD & 19.0 & 21.4 & \\
Undergraduate students & & 35.5 \\
Mean & 17.6 & 11.3 & \\
SD & 14.3 & 15.7 & \\
\hline
\end{tabular}

different categories, namely, high-risk investments versus medium- and low-risk investments, even when investment labels were absent. The empirical evidence shows us that 9 out of 15 bank customers $(60 \%)$ made significant inconsistencies, that is, they invested in much riskier portfolios than before and with asset allocations that deviated from the original ones, on average by $69 \%$ (calculated on the amount of the originally invested money). These results give us a perspective on how people perceive, represent, and act upon financial information and reveal a delicate aspect for potential manipulation of decisions through branding, for better or for worse. The inconsistency may have to do with some customers relying on the recognition heuristic in Task 3 , while this was not possible in Task 4 . The results confirm the role of the recognition heuristic: when it is not applicable, customers perform differently. ${ }^{4}$

Students' data confirm such conclusion although they recognized different investments: the weight on risky assets in this case increases when labels are provided, as shown by average values in Table 7 . Similarly, the average percentage change in portfolio composition when labels are removed is $30 \%$, much lower than the $69 \%$ observed in the bank customers sample. The reason for such differences across samples and for the different allocation across treatments in both subjects' samples is due to the familiarity with labels and to the recognition heuristic. In fact we know that students are only used to bank accounts, exploited for liquidity management, and presumably know that high gains can be obtained by stocks. When labels are at disposal, students thus move investments from bank accounts, which if labeled seem less attractive, to stocks. But students are less familiar with the proposed investments, and thus the average impact of labels is lower.

\section{Discussion}

The aim of this research was to investigate how average investors make financial decisions. We focused on two components of investor behavior: information search

\footnotetext{
${ }^{4}$ By recognition heuristic, we mean a simple strategy that allows individuals to infer, for example, which of two objects has a higher value on some numerical criterion, based on the fact that one is recognized and the other is not (e.g., predicting which investment has a higher expected return based on whether one recognizes the name of one of the shares). The recognition heuristic for such tasks is simply stated: If one of the two objects is recognized and the other is not, then infer that the recognized object has the higher value (Goldstein and Gigerenzer 2002).
} 
and decision process. We interviewed 80 customers of an Italian mutual bank, and we invited 15 of them to participate in an interactive experiment with four investment tasks. We designed the experimental setup to reflect as realistically as possible the actual decisions that take place in transactions between financial advisors and their customers. To a large extent, we succeeded in presenting information to customers that matched the information they typically encounter when making real financial decisions. We then applied the same experimental design to 56 young university students in order to test whether the results we obtained with bank customers replicate and to start to evaluate the possible role played by experience in investment choices.

In Task 1, we asked our subjects to choose between two investments while facing a strict information budget that limited information look-ups. In Task 2, subjects faced more investment possibilities and information space. We observed in Task 2 that subjects looked up less than half of the available investment features even when they were free to have more information and could have accessed all of it within a few additional seconds of search. More than half the bank customers subjects $(57 \%)$ looked up only a small set of non-overlapping features even when they were free to access the entire information set and it was feasible to do so with very modest time costs.

Although one of the samples size is small (in part, due to our bank partner's indication that our data collection had continued long enough), the rich combination of qualitative interview data from 80 respondents, information search data, and investment decision data reveal new empirical insight into information search and investment decision processes among real investors. Moreover the use of a larger sample of inexperienced investors further strengthens our results.

The evidence we presented suggests that investors usually consider only a strict subset of available information about the set of investment alternatives from which they choose, even when the universe of investments and related information sets is relatively small and search costs minimal. In this sense, investors appear to follow a heuristic search intuition that one finds as a key feature in a number of investment heuristics recently appearing in the behavioral finance literature (DeMiguel et al. 2009; Gigerenzer 2008).

Of particular interest was how infrequently subjects undertook pairwise comparisons that we commonly assume take place in order to completely rank one's choice set (e.g., the expected return of investment A and the expected return of investment B). Pairwise comparison of product features is the essential behavioral assumption underlying the rational choice model in economics and the preponderance of consumer choice models used in marketing research. And yet our evidence directly contradicts this fundamental assumption about consumers' decision process. Investors thus neither look at all available information neither they fully compare the available choices, and that is true for both experienced but non expert investors such as our bank customers and for completely inexperienced investors such as our university students.

Relying on the analysis of the behavior we observed, we modeled binary investment choice with a lexicographic decision tree that predicts $78 \%$ of choices correctly in the case of bank customers, and $74 \%$ of choices when university 
students are considered. The decision tree orders risk first among various investment features. That means that risk is more important that all other features combined. The decision tree model then uses a tallying rule on the remaining investment features.

Tallying is cognitively less demanding than weighted additive models, because it depends only on counting up pros and cons rather than computing weighted sums. We compared the predictive performance of these models against a competing linear model based on full-information utility maximization. The performance of the regression model predicts the choice data slightly better than simpler models, with 80 versus $78 \%$ predictive accuracy when analyzing bank customers data, but it looses its better accuracy when considering our second sample of university students. Moreover, in terms of model complexity, it costs many more parameters, which implies a large risk of overfitting. Finally, the regressed model ignores several information pieces and gives more weight on the information we selected as more relevant in the heuristic model. We also tried to compare the predictive accuracy of rational choice models although our experimental design does not allow an extensive comparison: the poor results we obtained strengthen our doubts about the possibility to accept rational choice models as empirically valid in investment decisions.

The second part of our experiment focused on portfolio choice. We discovered in Task 3 and 4 that when labels are missing, bank customers tend to select a riskier mix of investments than was selected when investment labels were available. On the contrary, undergraduate students showed smaller changes in portfolio composition and towards less risky investments, probably due to their different familiarity with investment labels. This suggests that seemingly superficial differences in the naming of two financial products with identical mean and variance could play a significant role in investment decisions. The recognition heuristic theory suggests how labelversus no-label tasks generated differences in observed choice behavior, although the informative role of investment names still needs more attention in future research as well as the impact of experience in investments does.

Financial products are presented to potential investors in the real world with a rapidly proliferating array of such labels. The names of things in the real-world investing environment very likely influence investor behavior, even though it should not according to standard portfolio choice theory. According to that theory, so long as the mean and standard deviation of all risky returns (including correlations, and possibly all higher moments for risk preference specifications more complicated than mean-variance preferences) are presented to the investor, his or her decisions should not be influenced by the order of presentation or names and labels applied to investment features. Behavior should be especially invariant to labeling changes that do not affect the first and second moments of random returns.

The heuristic model we tested suggests that subjects in our study rely on simple decision trees in which risk is, by far, the most important investment feature and non-compensatory. Furthermore, normative assessment of the performance of real bank customers' decision processes (relative to the neoclassical benchmark) indicates heuristic strategies appear to serve investors reasonably well. Whereas the biases and heuristics literature frequently assigns an automatic negative normative value to any decision procedure that deviates from the neoclassical ideal, we identify 
attractive normative properties of the heuristic approach (similar to the normative assessments in Magni 2009). Similar lexicographic decision-tree heuristics such as Gigerenzer and Goldstein's (1996) take-the-best heuristic consider the features of investments sequentially in a ranking determined by some measure of pairwise correlation or univariate predictive accuracy (rather than considering all their intercorrelatedness with other regressors in the model as, for example, partial correlations do). This helps reduce the cognitive processing required to execute heuristics and can improve robustness and accuracy of predictions (Gigerenzer and Brighton 2009).

Although it remains an open question as to what extent bank customers are able to judge the quality of their decisions in terms of outcomes and of processes, we noticed that our subjects clearly tended to consider specific combinations of investment features, and use these combinations according to predictable rules, even though they lacked theories on causal links between features and decision criteria. This evidence is made sound by its confirmation in the choices of completely inexperienced investors, although we do not know yet the reasons for such a pervasive behavior.

The tasks that subjects faced required them to, in some treatments, search freely for information, unlike most experimental economics tasks of financial decisions in which subjects are provided with a complete set of summary statistics (namely, expected values, variances, and covariances), which are required by standard models such as CAPM. The subjects in this study could decide how much information they wanted to look at. Most chose to look up some quantity of information less than the maximum of all that was available. And a significant proportion explored only a very small subset of overlapping investment features (i.e., looking up the same piece of information more than once). Subjects exhibited remarkably similar information search behavior across trials and samples, and their subjective reports about their search behavior coincided remarkably in that nearly all reported spending very little time in deliberation (and never calculation) in choosing portfolio weights. Moreover, subjects indicated that, although they were handling meaningful amounts of money, they nevertheless made investment decisions with relatively little cognitive effort in both lab experiments and in actual investment decision making. Information search was characterized by frugality and simplicity.

Given the crisis we are living through at the time of writing, and the accumulating evidence of mismatch between models of omniscient investors and the badly designed institutions that result from those models, we believe that behavioral research can play an important and positive role in improving risk communication and deepening the relationship between firms selling investment advice and their customers. We hope that improvements in descriptive models of investors' use of information and decision processes will, in turn, improve both the investment environment that average financial consumers face and the organization of financial institutions. Insofar as researchers discover better descriptive information about consumers' information needs and decisions processes, advancement toward satisfaction with the investment experience and with firms' and policy makers' ability to predict consumer behavior becomes more likely. Far from a zerosum game, there appears to be room for substantial improvements, from the vantage 
points of all parties, in the processes by which consumer-level financial transactions take place.

Acknowledgments We thank John Payne for very helpful comments and Michela Balconi for her ideas regarding data analysis. We also thank Davide Donati, general director of the Cassa Rurale Giudicarie Valsabbia Paganella, and all the Board of Executives, for their full support in conducting on-site research at their banks. We likewise thank Marcel Jentsch for valuable help programming and designing interactive interfaces. Riccardo Boero acknowledges financial support from Regione Piemonte [IIINBEMA Research Project].

\section{Appendix A}

The experimental design is driven by the principle of preserving the comparability with results obtained with bank customers creating a realistic investment environment.

It relies on the computation of payoffs for each choice made by subjects through the modeling of the outcome of each investment in a virtual financial market with realistic economic incentives and uncertainty.

Economic earnings are computed using experimental currency units (ECU) which are then converted in euro according to a fixed conversion rate that is explained above in the paper. In other words, in the following we talk about earnings in ECU but they correspond to actual money.

The payoff structure we develop is built as a unique payoff function which works for every investment because every single different feature of investments is represented by different parameters in the function. Below each element of the payoff function is singularly discussed, and finally some examples of the aggregated payoff function are presented.

The portfolio duration

Due to the aim of modeling the intrinsic uncertainty of investment decisions we rely on the happening of probabilistic events. In particular after each investment decision a time of duration of the portfolio (in Task 1 and 2 is the duration of the single investment that has been chosen because the investment portfolio collapses in the choice of a single investment) is randomly chosen, with a uniform probability distribution, among the five possible ones (i.e., immediate, short, medium and long, and very long that is to say, in numbers, $0,1,2,3$, and 4 ). The reason for considering the possibility of very short (i.e., "immediate") duration of investment decisions is to make even short duration investments subjects to some uncertainty as it will be better detailed below. Thus, the portfolio duration is a discrete random variable as follows:

$$
P D_{t}=\xi \in\{0,1,2,3,4\} ;
$$

where $P D_{t}$, the duration of the portfolio choice, is a random variable uniformly draw from the set of integers ranging from 0 to 4 (i.e., from very short to long).

The portfolio duration can be conceived as the time when the investor is asked to dismiss her portfolio due to an external shock (e.g., the need for liquidity for 
relocating, for medical care, etc....). It is an uncertain and random event that every investor knows to potentially face and that in our design models the uncertain lasting of investment decisions made by subjects.

A portfolio duration is, during the experiment, randomly drawn for each investment choice made by each subject: that is to say that it is randomly chosen for each subject on every repetition of each single task of the experiment and, obviously, it is not known by subjects before the choice.

\section{The payoff function}

The payoff function consider all the six characteristics differentiating investment decisions and the values each feature can take. In this paragraph we consider each feature and transform it to an ECUs value. The payoff function will then be just the sum of the ECUs obtained by each feature.

The characteristics of investment decisions that subjects face in the different experiment tasks are six and each one has a different set of possible values, as follows (the possible values of each feature are presented between brackets):

1. liquidity (easy, difficult);

2. coupon (no, yes);

3. cost before redemption (no, yes);

4. risk (low, medium, high);

5. time horizon (short, medium, long);

6. cost (low, medium, high).

The same list, represented in formal means is as follows (where "i" stands for each investment):
1. $\quad l i q_{i} \in\{0,1\}$
2. $\operatorname{cou}_{i} \in\{0,1\}$;
3. $c b r_{i} \in\{0,1\}$;
4. risk $_{i} \in\{0,1,2\}$;
5. $t i h_{i} \in\{0,1,2\}$;
6. cost $_{i} \in\{0,1,2\}$.

Liquidity The lack of liquidity of an investment can be a cost if the investor has to dismiss the investment before its natural end. Thus, such a cost emerges if and only if the chosen investment has "difficult" value of liquidity and the portfolio duration is shorter than the investment time horizon. In mathematical language liquidity contributes to the payoff with - urgency $_{t i} \cdot l i q_{i} \cdot k_{\text {liquidity }}$,

where urgency $_{i t}=\left\{\begin{array}{l}0, \text { when } t i h_{i}<P D_{t} \\ 1, \text { when } t i h_{i} \geq P D_{t}\end{array}\right.$, and that is to say that the contribution of the liquidity feature is a cost (a negative impact of the parameter $k_{\text {liquidity }}$ ) that happens only if the liquidity is difficult and the duration of the portfolio $\left(P D_{t}\right)$ is shorter than the time horizon of the investment $\left(t i h_{i}\right)$. 
Coupon The presence of coupons increases the return of an investment. Obviously it also depends on the time an investment is kept, and thus the amount of earnings deriving from coupons depends on the portfolio duration. In a formal way it is $P D_{t} \cdot \mathrm{Cou}_{i} \cdot k_{\text {coupon }}$, meaning that coupons (if present) increase the return of an investment by a constant value $\left(k_{\text {coupon }}\right)$ multiplied for the portfolio duration $\left(P D_{t}\right)$.

Cost before redemption The cost before redemption negatively modifies the return of an investment if and only if that feature characterizes the investment and if the portfolio duration is shorter than the investment time horizon (i.e., the urgency $_{t i}$ value computed as above equals 1$)$ : - urgency $_{t i} \cdot c b r_{i} \cdot k_{\text {costBeforeRedemption }}$.

Risk Risk is the main component of an investment return. If risk is higher, profits can be higher but also more uncertain. We combine different risk values (probabilities) and different returns promised by the investment. We thus extract a new, continuous and uniform, random variable "risk probability" $R P_{t i}=\xi \in$ $\left(\frac{3=r i s k_{i}}{3}, 1+r i s k_{i}\right]$ and multiply it for the parameter value $k_{\text {risk }}$.

The mathematical expression of the positive return coming from investments is thus $R P_{t i} k_{r i s k}$ which implies, according to risk $k_{i}$ values, the following maximum, average and minimum yields (Table 8).

As the table points out, riskier investments on average yield higher profits, but there is also the chance of gaining much less profits.

Time horizon The time horizon of the investment does not imply a cost per se but it affects other cost elements (i.e., liquidity and cost before redemption) impacting on the conditional variable called urgency $_{t i}$.

Cost The cost for buying an investment can vary and it represents a value decreasing the payoff of each investment. It is computed as $-\left(\operatorname{cost}_{i}+1\right) \cdot k_{\text {cost }}$, that is to say that the cost increases proportionally to the value of $\operatorname{cost}_{i}$ and to the parameter $k_{\text {cost }}$.

The payoff function Putting together the elements just introduced, the payoff function of an investment $i$ at time $t$ takes the following form:

$$
\begin{aligned}
\pi_{i t}= & R P_{i t} \cdot k_{\text {risk }}+P D_{t} \cdot \text { cou }_{i} \cdot k_{\text {coupon }}-\text { urgency }_{t i} \cdot \text { liq }_{i} \cdot k_{\text {liquidity }}-\text { urgency }_{t i} \cdot \text { cbr }_{i} \\
& \cdot k_{\text {costBeforeRedemption }}-\left(\text { cost }_{t}+1\right) \cdot k_{\text {cost }}
\end{aligned}
$$

where $\quad P D_{t}=\xi \in\{0,1,2,3,4\}, \quad$ urgency $_{i t}=\left\{\begin{array}{l}0, \text { when } t i h_{i}<P D_{t}, \\ 1, \text { when } \quad t i h_{i} \geq P D_{t},\end{array} \quad\right.$ and $R P_{t i}=\xi \in\left(\frac{3=r i s k_{i}}{3}, 1+\right.$ risk $\left._{i}\right]$.

Examples Just as an example to make the payoff computation clearer, it is possible to specify parameters values and to report some investment profits. 
Table 8 Yields according to different risks

\begin{tabular}{llll}
\hline & $\begin{array}{l}\text { Low risk investments } \\
\text { risk }\end{array}=0$ & $\begin{array}{l}\text { Medium risk investments } \\
\text { risk }_{i}=1\end{array}$ & $\begin{array}{l}\text { High risk investments } \\
\text { risk }_{i}=2\end{array}$ \\
\hline Minimum yield & $k_{\text {risk }}$ & $0.667 k_{\text {risk }}$ & $0.334 k_{\text {risk }}$ \\
Average (expected) yield & $k_{\text {risk }}$ & $1.334 k_{\text {risk }}$ & $1.667 k_{\text {risk }}$ \\
Maximum yield & $k_{\text {risk }}$ & $2 k_{\text {risk }}$ & $3 k_{\text {risk }}$ \\
\hline
\end{tabular}

\section{Hypotheses}

- Parameters values: $k_{\text {risk }}=100, k_{\text {coupon }}=10, k_{\text {liquidity }}=10, k_{\text {costBeforeRedemp- }}$ tion $=10, k_{\text {cost }}=5$.

- Payoff function:

$$
\begin{aligned}
\pi_{i t}= & R P_{i t} \cdot 100+P D_{t} \cdot \text { cou }_{i} \cdot 10-\text { urgency }_{t i} \cdot \text { liq }_{i} \cdot 10-\text { urgency }_{t i} \cdot \text { cbr }_{i} \cdot 10 \\
& -\left(\text { cost }_{t}+1\right) \cdot 5
\end{aligned}
$$

1. Insurance with guaranteed capital:

- Features: liquidity $=$ difficult, coupon $=$ no, cost before redemption $=$ yes, risk $=$ low, time horizon $=$ medium, cost $=$ high .

- Randomly drawn variables: $P D_{t}=1$, urgency $_{t i}=1$ (in fact time horizon is medium and $P D_{t}$ is short), $R P_{t i}=1$.

- The payoff: $\pi_{i t}=1 \cdot 100+1 \cdot 0 \cdot 10-1 \cdot 1 \cdot 10-1 \cdot 1 \cdot 10-(2+1) \cdot 5=$ 65.The result is that an insurance investment, if dismissed before its natural end, generate lower earnings.

2. Government Bonds:

- Features: liquidity $=$ easy, coupon $=$ yes, cost before redemption $=$ yes, risk $=$ low, time horizon $=$ medium, cost $=$ low .

- Randomly drawn variables: $P D_{t}=3$, urgency $_{t i}=0$ (in fact time horizon is medium and $P D_{t}$ is long), $R P_{t i}=1$.

- The payoff: $\pi_{i t}=1 \cdot 100+3 \cdot 1 \cdot 10-0 \cdot 0 \cdot 10-0 \cdot 1 \cdot 10-(0+1) \cdot 5$ $=125$. The result is that the investment in government bonds is hre prolonged and coupon earnings are obtained for the duration of the investment.

Parameters values During the experiment, the parameters used for calculating subjects' profits are given in Table 9.

\section{Appendix B}

Overlapping Information Index

Give a set of information for investment 1 , denoted A, and a set of information for investment 2 , denoted $\mathrm{B}$, which contain all the information explored by each subject 
Table 9 Parameters used in the experiment

$k$ parameters are the ones just
described while Exploration cost
is the cost of unveiling the value
of an investment feature

\begin{tabular}{ll}
\hline Parameter & Value (in ECU) \\
\hline Exploration cost & 6 in Task 1, 2 in Task 2 \\
$k_{\text {risk }}$ & 100 \\
$k_{\text {coupon }}$ & 5 \\
$k_{\text {liquidity }}$ & 10 \\
$k_{\text {costBeforeRedemption }}$ & 10 \\
$k_{\text {cost }}$ & 20 \\
\hline
\end{tabular}

for each investment choice, the OII measure of overlapping information (introduced in the body of this paper) is simply the cardinality of the intersection of these two sets for each participant:

$$
O I I=|A \cap B|
$$

\section{Order Preservation Index}

The Order Preserving Index takes not of the order in which the elements of the information sets A and B were generated by subjects' search behavior. We write the elements of the set $A$ as a vector that records this sequence: $a=\left[a_{1}, a_{2}, \ldots, a_{n}\right]$, where $\mathrm{n}$ denotes the number of items searched for or looked up by a particular subject. The index of subjects' identities is suppressed here for expositional clarity in describing the information indexes used in the paper's section on process tracing. Thus, $a_{i}$ was looked up before $a_{j}$ if and only if $i<j$. Similarly, a vectorized version of the set $\mathrm{B}$ that records the order in which information was looked up is denoted as $\mathrm{b}=\left[\mathrm{b}_{1}, \mathrm{~b}_{2}, \ldots, \mathrm{b}_{\mathrm{n}}\right]$.

The $(n+1) \times(m+1)$ matrix referred to as the so-called F matrix (see Payne et al. 2004) plays a key role in process tracing. The first column and first row of the matrix $\mathrm{F}$ are initialized as 0 . In MATLAB command language, this is expressed as $\mathrm{F}(1,1: \mathrm{m}+1)=0$ and $\mathrm{F}(1: \mathrm{n}+1,1)=0$.

The rest of the matrix is computed according to a recurrence relation, the Needleman-Wunsch algorithm. The order of the steps follow from the sequence given by vectors $a$ and $b$.

$$
F(i, j)=\max \left\{\begin{array}{l}
F(i-1, j-1)+s\left(a_{i-1}, b_{j-1}\right) \\
F(i-1, j) \\
F(i, j-1)
\end{array}\right.
$$

where

$$
s\left(a_{i}, b_{j}\right)= \begin{cases}1, & \text { if } a_{i}=b_{j} \\ 0, & \text { otherwise }\end{cases}
$$

Finally, the OPI is computed as:

$$
\mathrm{OPI}=\left(100\left(\frac{F(n+1, m+1)}{\max (n, m)}\right)\right) \%
$$


Fig. 6 Example of computation of the order preserving index

\begin{tabular}{cc|ccc}
\hline & & $R$ & $L$ & $C$ \\
\hline & 0 & 0 & 0 & 0 \\
\hline$C$ & 0 & 0 & 0 & 1 \\
\hline$R$ & 0 & 1 & $1 \longrightarrow 1$ \\
\hline$L$ & 0 & 1 & $2 \longrightarrow 2$ \\
\hline
\end{tabular}

The expression above is either normalized to a $0-100$ percentage-point scale or interpreted as decimal representations of percentages that lie in the unit interval. An example that visually illustrates the recursion relation described above symbolically follows. This visual corresponds to the example discussed in. Sect. 3.4 (Fig. 6)

\section{References}

Balzer WK, Doherty ME, O'Connor R (1989) Effects of cognitive feedback on performance. Psychol Bull 106(3):410-433

Berg N, Hoffrage U (2008) Rational ignoring with unbounded cognitive capacity. J Econ Psychol 29(6):792-809

Chewning E, Harrell A (1990) The effect of information overload on decision makers' cue utilization levels and decision quality in financial distress decision task. Acc Org Soc 15(6):527-542

DeMiguel V, Garlappi L, Uppal R (2009) Optimal versus naive diversification: how inefficient is the $1 / \mathrm{N}$ portfolio strategy. Rev Financ Stud 22:1915-1953

Fischbacher U (2007) z-Tree: zurich toolbox for ready-made economic experiments. Exp Econ 10(2):171-178

Gigerenzer G (2008) Why heuristics work. Perspect Psychol Sci 3:20-29

Gigerenzer G, Brighton H (2009) Homo heuristicus: why biased minds make better inferences. Topics Cogn Sci 1:107-143

Gigerenzer G, Goldstein DG (1996) Reasoning the fast and frugal way: models of bounded rationality. Psychol Rev 103(4):650-669

Gigerenzer G, Todd PM, the ABC Group (1999) Simple heuristics that make us smart. Oxford University Press, New York

Goldstein DG, Gigerenzer G (2002) Models of ecological rationality: the recognition heuristic. Psychol Rev 109:75-90

Lee BK, Lee WN (2004) The effect of information overload on consumer choice quality in an on-line environment. Psychol Market 21:159-183

Loewenstein G (2006) The pleasures and pains of information. Science 312:704-706

Lynch JG Jr, Ariely D (2000) Wine online: search costs and competition on price, quality, and distribution. Market Sci 19:83-103

Magni CA (2009) Investment decisions, net present value and bounded rationality. Quant Financ 9(8):967-979

Martignon L, Katsikopoulos KV, Woike JK (2008) Categorization with limited resources: a family of simple heuristics. J Math Psychol 52:352-361

Martignon L, Laskey K (1999) Bayesian benchmarks for fast and frugal heuristics. In: Gigerenzer G, Todd PM, the ABC Group (eds) Simple heuristics that make us smart. Oxford University Press, New York

Payne J, Bettman R, Johnson EG (2004) Fast and frugal heuristics: the tools of bounded rationality. In: Koehler D, Harvey N (eds) Handbook of judgment and decision-making. Blackwell, Oxford, pp 62-89

Train KE (2003) Discrete choice methods with simulation. Cambridge University Press, Cambridge

Yee M, Dahan E, Hauser JR, Orlin JB (2007) Greedoid-based non-compensatory two-stage considerationthen-choice inference. Market Sci 26:532-549 